\title{
Article
}

\section{Resveratrol prevents social deficits in animal model of autism induced by valproic acid}

Bambini-Junior, Victorio, Zanatta, Geancarlo, Della Flora Nunes, Gustavo, Mueller de Melo, Gabriela, Michels, Marcus, Fontes-Dutra, Mellanie, Nogueira Freire, Valder, Riesgo, Rudimar and Gottfried, Carmem

Available at http://clok.uclan.ac.uk/18494/

Bambini-Junior, Victorio ORCID: 0000-0002-8590-6770, Zanatta, Geancarlo, Della Flora Nunes, Gustavo, Mueller de Melo, Gabriela, Michels, Marcus, Fontes-Dutra, Mellanie, Nogueira Freire, Valder, Riesgo, Rudimar and Gottfried, Carmem (2014) Resveratrol prevents social deficits in animal model of autism induced by valproic acid. Neuroscience Letters, 583 . pp. 176-181. ISSN 0304-3940

It is advisable to refer to the publisher's version if you intend to cite from the work. http://dx.doi.org/10.1016/j.neulet.2014.09.039

For more information about UCLan's research in this area go to http://www.uclan.ac.uk/researchgroups/ and search for < name of research Group>.

For information about Research generally at UCLan please go to http://www.uclan.ac.uk/research/

All outputs in CLoK are protected by Intellectual Property Rights law, including Copyright law. Copyright, IPR and Moral Rights for the works on this site are retained by the individual authors and/or other copyright owners. Terms and conditions for use of this material are defined in the policies page. 


\section{RESVERATROL PREVENTS AUTISTIC-LIKE SOCIAL DEFICITS IN THE ANIMAL MODEL OF AUTISM INDUCED BY VALPROIC ACID}

Victorio Bambini-Junior $^{1,2^{*}}$, Geancarlo Zanatta ${ }^{1,2}$, Gustavo Della Flora Nunes ${ }^{1,2}$, Gabriela Mueller de Melo ${ }^{1,2}$, Marcus Michels ${ }^{1,2}$, Mellanie Dutra ${ }^{1,2}$, Valder Nogueira Freire $^{4}$, Rudimar Riesgo ${ }^{2,3}$, Carmem Gottfried ${ }^{1,2}$

FEDERAL UNIVERSITY OF RIO GRANDE DO SUL, PORTO ALEGRE, RS, BRAZIL: ${ }^{1}$ Research Group in Neuroglial Plasticity at the Department of Biochemistry, Institute of Health's Basic Science. ${ }^{2}$ Translational Research Group in Autism Spectrum Disorders (GETTEA). ${ }^{3}$ Child Neurology Unit, Clinical Hospital of Porto Alegre (HCPA).

FEDERAL UNIVERSITY OF CEARA, FORTALEZA, CE, BRAZIL: ${ }^{4}$ Department of Physics.

*CORRESPONDENCE ADDRESS:

Victorio Bambini-Junior

Departamento de Bioquímica

Instituto de Ciências Básicas da Saúde

Universidade Federal do Rio Grande do Sul

Rua Ramiro Barcelos 2600 anexo

90035 - 003 - Porto Alegre, RS - BRAZIL

Fax: +55 5133085540 Phone: +55 5133085565

E-mail: victoriobambini@gmail.com 


\section{Abstract}

Autism spectrum disorders (ASD) involve a complex interplay of both genetic and environmental risk factors, such as prenatal exposure to valproic acid (VPA). Considering the neuroprotective, antioxidant and anti-inflammatory effects of resveratrol (RSV), we investigated the influence of prenatal RSV treatment on social behaviors of a rodent model of autism induced by prenatal exposure to VPA. In the three-chambered apparatus test, the VPA group showed a reduced place preference conditioned by conspecific and no preference between exploring a wirecage or a rat enclosed inside a wire cage, revealing sociability impairments. Prenatal administration of RSV prevented the VPA-induced social impairments evaluated in this study. A bioinformatics analysis was used to discard possible molecular interactions between VPA and RSV during administration. The interaction energy between RSV and VPA is weak and highly unstable, suggesting cellular effects instead of a single chemical process. In summary, the present study highlights a promising experimental strategy to evaluate new molecular targets possibly involved in the etiology of autism and developmental alterations implicated in neural and behavioral impairments in ASD.

Keywords: Autism spectrum disorder, valproate, resveratrol, animal model, behavior. 


\section{Abbreviations}

ASD - Autism Spectrum Disorders

DFT - Density Functional Theory

KRG - Korean Red Ginseng

RSV - Resveratrol

SI - Sociability Index in the Three-Chamber Test

$\mathrm{SNI}$ - Social Novelty Preference Index in the Three-Chamber Test

VPA - Valproic Acid

MD - Molecular Dynamics

\section{Introduction}

Autism spectrum disorders (ASD) comprise a set of developmental disabilities characterized by social impairments, communication difficulties, and restricted and stereotyped patterns of behavior [19]. This group of disorders is attracting great public attention because of their high prevalence, elevated social cost and large impact on the family. The US Center for Disease Control and Prevention estimate the prevalence of autism in the United States to be 1 in 68 children, with boys 4.5 fold more affected than girls [1].

In 1943, a landmark paper describing autism was published by Leo Kanner [11]; however, even after 70 years, the etiology of autism and its molecular basis are not well understood. Genetic studies have revealed a multitude of alterations associated with autism, but the characterized components to date account for only $25 \%$ of all cases of autism [17]. On the other hand, environmental factors, like exposure to 
xenobiotics (e.g. valproic acid (VPA) and thalidomide) can either trigger or contribute to autism development [8].

Considering the association between VPA exposure and ASD in humans [4], an animal model of prenatal VPA administration in rodents was suggested. In the past years, this model has shown to be a reliable research tool, as it presents many morphological and behavioral alterations related to the autism pathophysiology [3, 5]. Thus, prevention of complex outcomes, such as behavioral impairments, and investigation of the molecular pathways that underlie these changes in the VPA model can shed light in biological process relevant to autism etiology. Resveratrol (RSV) is a naturally-occurring polyphenolic compound present in grapes, pines, peanuts and red wine [25]. The bulk of an intravenous dose of RSV is mainly converted to sulphate conjugates within approximately 30 minutes in humans and the serum half-life of total RSV metabolites is approximately 9.2 hours [26]. In the last two decades, RSV received special attention from scientific community and has been associated with protective and therapeutic roles in several illnesses [25]Resveratrol is widely recognized as an anti-oxidant and antiinflammatory compound besides showing neuroprotective effects [7]. All of these biological activities could be of interest in autism therapeutics [21].

In this context, we investigated preventive effects of RSV in the autistic-like social features of an animal model induced by prenatal exposure to VPA. We performed a three-chamber test to measure social memory and preferences. Additionally, bioinformatics studies were used to evaluate the interaction between VPA and RSV, in order to distinguish whether the effect derivate from a direct molecule-molecule contact or from a broad cellular action. 


\section{Results}

\subsection{Behavioral testing}

\subsubsection{Three-chamber sociability and social novelty test}

\subsubsection{Sociability test:}

Animals of all groups stayed in the central chamber (known chamber) for only short periods of time. The Control rats spent significantly more time in the chamber with the conspecific than in the chamber with the object (mean \pm SEM: $285.14 \pm 9.55$ and $224.86 \pm 12.24$, respectively; $p<0.001)$. In contrast, VPA-exposed rats showed no preference between these chambers. Interestingly, RSV treatment was able to prevent the change induced by VPA (RSV+VPA: $289.5 \pm 10.56$ and $216.3 \pm 21.26$; $p<0.05)$. Rats from RSV group behaved similarly to the Control animals, but there was no statistical difference between the times spent in the chamber with the rat and the chamber with the object. These data show that the VPA group animals could be avoiding social contact, an atypical behavioral pattern that was prevented by RSV prenatal treatment (Figure $1 \mathrm{~A}$ ).

The analysis of exploration time, defined as the period in which the rat being tested is near the enclosed rat or the object and actively interacting with it (Figure 1B), shows that rodents from both Control and RSV groups preferentially interact with a conspecific than an object (Control: $232.29 \pm 10.42$ and $117.43 \pm 11.67 ; p<0.001$. RSV: $247.43 \pm 38.8$ and $91.57 \pm 19.01 ; p<0.05$. Rat and object, respectively). The VPA animals showed no difference between the contact times with the novel rat and the object (147.17 \pm 21.54 and $189.44 \pm 25.11)$, which clearly shows decreased sociability. The RSV treatment was again able to counteract the VPA effect $(214.5 \pm 9.02$ and $112.4 \pm 11.43 ; p<0.001)$. 
Animals from Control and RSV groups showed no difference in the Sociability Index (SI). However, it is significantly reduced in the VPA animals compared to the Control $(-0.083 \pm 0.127$ and $0.337 \pm 0.046$, respectively; $p<0.05)$. The VPA+RSV group SI $(0.319 \pm 0.066)$ was similar to the Control and showed a trend to be different from the VPA group (Figure 1C).

\subsubsection{Social novelty test}

Animals of all groups spent less time in the central chamber than in other chambers (Figure 2A). The Control animals preferred the chamber with the known rat to the chamber with the novel rat $2(127.14 \pm 32.32$ and $429.14 \pm 34.35$; $p<0.001)$, indicating an interest in social novelty and/or the formation of social memory. The VPA-exposed rats did not show difference in the time spent in each chamber while VPA+RSV group showed a behavioral pattern similar to the Control group, although the result was not statistically significant. The RSV group animals tended to spend more time in the chamber with the novel rat 2.

As observed in Figure 2B, Control $(80 \pm 19.19$ and $213.29 \pm 14.73 ; p<0.001)$ and RSV (127.14 \pm 19.14 and $210.86 \pm 28.04 ; p<0.05)$ group animals explored the known rat significantly less than the novel rat 2 . On the other hand, the VPAexposed rodents explored both rats to the same extent. RSV once more was able to prevent this alteration ( $66 \pm 18.32$ and $174.22 \pm 24.52 ; p<0.01$ ), bringing the behavior back to the Control pattern in VPA+RSV group animals.

The Social Novelty Preference Index (SNI) was at same level in both Control and RSV groups. Nonetheless, there is a reduction in the VPA animals compared to the Control $(-0.004 \pm 0.073$ and $0.485 \pm 0.125$, respectively; $p<0.01)$. The RSV treatment brought the index back to the same statistical level of the Control in the 
VPA+RSV animals $(0.436 \pm 0.17)$, and was statistically different from the VPA group $(\mathrm{p}<0.05)$ (Figure $2 \mathrm{C})$.

\subsubsection{Raw data and multiple interactions analysis}

The following data are provided as supplementary online material: raw data (Table S1 and Table S2), multiple interactions of all factors for time in chamber and exploration time (Tables S3 and S4, respectively) and descriptive statistics of SI and SNI index (Table S5).

\subsection{In silico analyses:}

\subsubsection{Molecular dynamics}

Simulations in a cubic box of water through classical molecular dynamics allowed the observation of the VPA and resveratrol interaction during $5 \mathrm{~ns}$. Simulations were performed in duplicate and no significant differences were observed between them. Molecules moved freely through the box during the simulation and neither aggregates nor long term interactions were observed. As seen in Figure 3A, in the first simulation only three molecules of VPA moved closer to resveratrol in distinct times. Nevertheless, these interactions were unstable and molecules quickly moved away from each other, suggesting the inability of complex formation. Figure $3 \mathrm{~B}, \mathrm{C}$ and $\mathrm{D}$ are representative of distinct VPA molecules interaction with resveratrol during the simulation.

It is available as supplementary material: Description of VPA and RSV protonation state, optimized structures in vacuum and electron density analysis (Figure S1); molecular interactions between VPA and RSV molecules in vacuum and in a cubic water box containing $\mathrm{Na}^{+}$and $\mathrm{Cl}^{-}$as counter ions (Figure S2); and a table showing 
the calculated interaction energies between distinct VPA molecules and RSV (Table S6).

\section{Discussion}

One of the most crucial areas of ASD research is the role of environmental factors in the development of autism. The present data clearly shows that the development of autistic-like social behaviors induced by prenatal exposure to VPA was highly counteracted by RSV, indicating a promising subject to investigate etiological clues and molecular alterations.

It was recently reported that the prenatal treatment with Korean Red Ginseng (KRG) extract resulted in a dose-dependent improvement in social behavior and tail malformations in the VPA animal model of autism [14]. However, it is important to take into account that KRG extract is composed of several different molecules, hindering the investigation about the mechanisms whereby KRG extract improves these behavioral impairments. In a different study, VPA animals treated prenatally with bumetanide showed electrophysiological patterns and ultrasonic vocalizations similar to the Control group [23]. Nonetheless, the authors did not evaluate any social parameter in the offspring.

Aiming to verify if residual RSV in the blood stream could interact with VPA, a molecular dynamics study was carried out in a biological fluid. The rate RSV:VPA at the blood stream is supposed to be less than 1:272 (molecule:molecule). During simulations an extreme situation was imposed, using RSV:VPA at a rate of 10:270 (molecule:molecule). As expected, due to the physicochemical features of the molecules, it was observed some degree of interaction between VPA and RSV. 
Nevertheless, likely due to the effect of the solvent, interactions in a water box demonstrated to be highly unstable. In this way, we can conclude that RSV is having an indirect effect against prenatal exposure to VPA, probably acting at the cellular level.

There is no clear evidence of the molecular mechanisms by which VPA can trigger autism in humans or autistic-like features in the animal model. Although, its histone deacetylase (HDAC) inhibitory activity seems to be important to the effects [12]. On the other hand, RSV is largely believed to perform at least part of its actions by regulating the level and activation of sirtuins (members of the class-III HDAC) in a substrate-specific manner [15]. Thus, further research may investigate the overall epigenetic alterations triggered by RSV and VPA, searching for opposite effects. Interestingly, the tail kink characteristically present in the VPA-exposed animals was not prevented by RSV prenatal treatment. This may be initial evidence that the neural-tube defects triggered by VPA are not the main mechanism for the development of autistic-like characteristics by these animals.

Another promising topic to be studied is the well-established immune alterations involved in the pathophysiology of autism [2], also observed in the animal model induced by VPA [22].Since this model demonstrates aberrant inflammatory responses toward a peripheral inflammatory stimulus [16] and resveratrol exerts anti-inflammatory effects, future studies should analyze its developmental role in the immune system.

In order to advance the knowledge about ASD development, several lines of investigation must be pursued, including research that could merge targets of VPA and RSV to clarify molecules and pathways affected by both. In this respect, we 
anticipate that further understanding of these molecular targets will be relevant to both therapeutic and etiological aspects of ASD. Similarly, such studies will hopefully help to understand ASD-related epigenetic modulation and developmental alterations implicated in the neural and behavioral impairments.

\section{Experimental procedures}

\subsection{Ethics statement}

Experiments were performed according to the NIH Guide for the Care and Use of Laboratory Animals and approved by local authorities.

\subsection{Animals}

Female Wistar rats from the local breeding colony (ICBS-Federal University of Rio Grande do Sul), were kept with a 12:12 light cycle (lights on at 7:00 and lights off at 19:00), controlled temperature $\left(22 \pm 1^{\circ} \mathrm{C}\right)$. Animals were mated overnight and if in the morning spermatozoa were found in vaginal secretion, this day was designated as the first day of pregnancy. Females were kept separated and with free access to their own litters. The offspring was weaned at 21 days old and were housed separately by sex. Rats had free access to food and water. Only male animals were tested. All the experiments were conducted between 14:00 and 18:00. Behavioral tests were performed with 35-50 day old animals. We used at least 3 pregnant female Wistar rats per group, with litter sizes ranging from 3 to 12 animals. The three chambers test was performed for the control group $(n=7), R S V$ group ( $n=7)$, VPA group $(n=12-19)$ and VPA+RSV group $(n=9-10)$.

\subsection{Treatments}


Valproic Acid (Acros Organics, New Jersey, USA) was purchased as the sodium salt and dissolved in $0.9 \%$ saline for a concentration of $250 \mathrm{mg} / \mathrm{mL}$. Females received a single intraperitoneal injection of $600 \mathrm{mg} / \mathrm{kg}$ VPA or physiological saline on Embryonic day 12.5 (E12.5) [3].

Resveratrol (Fluxome, Stenløse, Denmark) was dissolved in DMSO (Merck, New Jersey, USA) for a concentration of $36 \mathrm{mg} / \mathrm{mL}$. Females received daily subcutaneous injections of $3.6 \mathrm{mg} / \mathrm{kg}$ of RSV solution or the correspondent volume of DMSO. This low dose was chosen due to: (1) the growing evidence of harmful effects of higher doses of RSV during gestation to the dams and to the offspring [20]; and (2) even very low doses are able to generate beneficial biological effects [10].On E6.5 the pregnant rats were randomly separated in 4 groups: Control (which received only DMSO injections); RSV (which received only RSV injections); VPA (which received DMSO plus VPA injection); and VPA+RSV (which received RSV plus VPA injection). Every day, in the afternoon, pregnant rats were weighed and the treatment was applied according to the groups. The treatment lasted 13 days (E6.5 until E18.5) for each group.

\subsection{Behavioral test: Three-chamber sociability and social novelty: We} performed a 5 minutes long habituation in the central chamber in the previous day and immediately before the Sociability Test and conducted the experiments as previously described [3].At the beginning, an object was positioned in one of the lateral chambers, and a set animal+object was placed in the opposite lateral chamber. This animal (called from now on, novel rat 1) was an experimentally naive male Wistar rat with no previous contact with the test animals. The object was an empty cage identical to the one used to enclose the novel rat 1 . Time spent 
in each chamber, as well as the time spent exploring the novel rat 1 or the novel object, was analyzed by two observers during $10 \mathrm{~min}$. Immediately after, the social novelty test began. In this test, the novel rat 1 remained in its wire cage (now it is called the known rat) and a new unfamiliar rat (novel rat 2) was placed in the wire cage in the opposite side (which was previously empty). Time spent in each chamber and time spent sniffing each wire cage was recorded during $10 \mathrm{~min}$. It is important to note that rats that did not explore all three chambers during the sociability test did not perform the social novelty test. In addition, since the social novelty test aims to evaluate the preference for social novelty and the formation of social memories, rodents that did not interact with both the known rat and the novel rat 2 were excluded from the analysis.

We also evaluated a Sociability Index (SI) (according to Kim et al. [13] and modified by us and R. Romcy-Pereira, personal communication), a mathematical transformation designed to allow the direct comparison of social behavior of the groups. It ranges from -1 to 1 and as the score becomes more positive and closer to 1, the more social the animal. The SI was calculated as showed below:

$$
S I=\frac{\text { Time exploring }_{\text {novel rat } 1}-\text { Time exploring } \text { novel object }_{\text {ime }}}{\text { Time exploring novel rat } 1+\text { Time exploring } \text { novel object }_{\text {im }}}
$$

In an analogous manner, we can use a Social Novelty Preference Index (SNI). It also ranges from -1 to 1 and a value closer to 1 indicates an animal more interested in social novelty. The SNI is calculated as showed below: 


$$
S I=\frac{\text { Time exploring }_{\text {novel rat } 1}-\text { Time exploring }_{\text {novel object }}}{\text { Time exploring }}
$$

\subsection{Bioinformatics: Molecular dynamics simulations and analysis}

Molecular dynamics (MD) simulations were performed with the package

GROMACS v. 4.5.1 [24], using the SPC water model and the GROMOS 53a6 force field [18]. A cubic box with edges of $12 \mathrm{~nm}$ (box volume $\approx 1728 \mathrm{~nm}^{3}$ ) containing RSV and VPA molecules in a proportion of 1:27 was built, and filled with 57.084 water molecules. An appropriate number of chloride and sodium counter-ions were added to neutralize the system, observing the final salt concentration of 0.15 mol.liter ${ }^{-1}$. Two independent 5 ns simulations were performed, differing only by the initial system coordinates: in the first (MD_1) the RSV molecule was placed in the center of the simulation box, while in the second (MD_2) it was randomly placed inside the box with the genbox software. The VMD program [9] was used to visualize and manipulate the system, and the g_mindist software was applied to calculate the minimum distance between the molecules during the simulation. Minimum distance plots were generated with XMGrace software (http://plasmagate.weizmann.ac.il/Grace/).

\subsection{Statistical analysis}

The values measured for the animals were integrated in a multivariate linear model to predict the impact of the treatment in the behavioral outcome. We used the Generalized Estimation Equations (GEE) in order to enable the comparison 
between multiple interdependent variables and overcome the necessity of normality and homoscedasticity. Bonferroni post-hoc test was used as the final evaluation. Sociability and Social Novelty Preference Indices were compared by Kruskal-Wallis test. Data is reported as mean \pm standard error of the mean (SEM). All analyses were performed using the SPSS program, Version 17.0 (SPSS, Chicago, IL).

Acknowledgements: This work was supported by the National Counsel of Technological and Scientific Development (CNPq), Coordination for the Improvement of Higher Education Personnel (CAPES), FINEP and INCT-EN National Institute of Science and Technology for Excitotoxicity and Neuroprotection. Special thanks to Janine MacKenzie for the careful English review. We would also like to thank Fluxome (Stenløse, Denmark) for the generous gift of resveratrol. 


\section{References}

[1] Center for Disease Control, Prevalence of Autism Spectrum Disorders Prevalence of Autism Spectrum Disorder Among Children Aged 8 Years - Autism and Developmental Disabilities Monitoring Network, 11 Sites, United States, 2010; Surveillance Summaries, March 28, 2014 / 63(SS02);1-21

[2] P. Ashwood, P. Krakowiak, I. Hertz-Picciotto, R. Hansen, I.N. Pessah, J. Van de Water, Associations of impaired behaviors with elevated plasma chemokines in autism spectrum disorders, Journal of neuroimmunology 232 (2011) 196-199.

[3] V. Bambini-Junior, L. Rodrigues, G.A. Behr, J.C. Moreira, R. Riesgo, C. Gottfried, Animal model of autism induced by prenatal exposure to valproate: Behavioral changes and liver parameters, Brain research 1408 (2011) 8-16.

[4] R.L. Bromley, G. Mawer, J. Clayton-Smith, G.A. Baker, Autism spectrum disorders following in utero exposure to antiepileptic drugs, Neurology 71 (2008) 1923-1924.

[5] D. Dufour-Rainfray, P. Vourc'h, A.M. Le Guisquet, L. Garreau, D. Ternant, S. Bodard, E. Jaumain, Z. Gulhan, C. Belzung, C.R. Andres, S. Chalon, D. Guilloteau, Behavior and serotonergic disorders in rats exposed prenatally to valproate: A model for autism, Neuroscience Letters 470 (2010) 55-59.

[6] E. Fombonne, The epidemiology of autism: a review, Psychol Med 29 (1999) 769 786.

[7] C. Gottfried, A. Quincozes-Santos, K. Basli, T. Richard, Resveratrol and Neuroprotection, Nova Publisher, 2011.

[8] M.R. Herbert, Contributions of the environment and environmentally vulnerable physiology to autism spectrum disorders, Current opinion in neurology 23 (2010) 103-110.

[9] W. Humphrey, A. Dalke, K. Schulten, VMD: visual molecular dynamics, Journal of molecular graphics 14 (1996) 33-38, 27-38.

[10] Y.H. Jing, K.H. Chen, P.C. Kuo, C.C. Pao, J.K. Chen, Neurodegeneration in streptozotocin-induced diabetic rats is attenuated by treatment with resveratrol, Neuroendocrinology 98 (2013) 116-127.

[11] L. Kanner, Autistic Disturbances of Affective Contact, Nervous Child (1943) 217250.

[12] S. Kataoka, K. Takuma, Y. Hara, Y. Maeda, Y. Ago, T. Matsuda, Autism-like behaviours with transient histone hyperacetylation in mice treated prenatally with valproic acid, The international journal of neuropsychopharmacology / official scientific journal of the Collegium Internationale Neuropsychopharmacologicum 16 (2013) 91-103.

[13] K.C. Kim, P. Kim, H.S. Go, C.S. Choi, S.I. Yang, J.H. Cheong, C.Y. Shin, K.H. Ko, The critical period of valproate exposure to induce autistic symptoms in Sprague-Dawley rats, Toxicology letters 201 (2011) 137-142.

[14] P. Kim, J.H. Park, K.J. Kwon, K.C. Kim, H.J. Kim, J.M. Lee, H.Y. Kim, S.H. Han, C.Y. Shin, Effects of Korean red ginseng extracts on neural tube defects and impairment of social interaction induced by prenatal exposure to valproic acid, Food Chem Toxicol 51 (2013) 288-296.

[15] M. Lakshminarasimhan, D. Rauh, M. Schutkowski, C. Steegborn, Sirt1 activation by resveratrol is substrate sequence-selective, Aging 5 (2013) 151-154. 
[16] L. Lucchina, A.M. Depino, Altered Peripheral and Central Inflammatory Responses in a Mouse Model of Autism, Autism research : official journal of the International Society for Autism Research (2013).

[17] J.H. Miles, Autism spectrum disorders--a genetics review, Genetics in medicine : official journal of the American College of Medical Genetics 13 (2011) 278-294.

[18] C. Oostenbrink, T.A. Soares, N.F. van der Vegt, W.F. van Gunsteren, Validation of the 53A6 GROMOS force field, European biophysics journal : EBJ 34 (2005) 273284.

[19] I. Rapin, R.F. Tuchman, Autism: definition, neurobiology, screening, diagnosis, Pediatr Clin North Am 55 (2008) 1129-1146, viii.

[20] V.H. Roberts, L.D. Pound, S.R. Thorn, M.B. Gillingham, K.L. Thornburg, J.E. Friedman, A.E. Frias, K.L. Grove, Beneficial and cautionary outcomes of resveratrol supplementation in pregnant nonhuman primates, FASEB journal : official publication of the Federation of American Societies for Experimental Biology 28 (2014) 2466-2477.

[21] D.A. Rossignol, R.E. Frye, Evidence linking oxidative stress, mitochondrial dysfunction, and inflammation in the brain of individuals with autism, Frontiers in physiology 5 (2014) 150.

[22] T. Schneider, A. Roman, A. Basta-Kaim, M. Kubera, B. Budziszewska, K. Schneider, R. Przewlocki, Gender-specific behavioral and immunological alterations in an animal model of autism induced by prenatal exposure to valproic acid, Psychoneuroendocrinology 33 (2008) 728-740.

[23] R. Tyzio, R. Nardou, D.C. Ferrari, T. Tsintsadze, A. Shahrokhi, S. Eftekhari, I. Khalilov, V. Tsintsadze, C. Brouchoud, G. Chazal, E. Lemonnier, N. Lozovaya, N. Burnashev, Y. Ben-Ari, Oxytocin-mediated GABA inhibition during delivery attenuates autism pathogenesis in rodent offspring, Science 343 (2014) 675-679.

[24] D. Van Der Spoel, E. Lindahl, B. Hess, G. Groenhof, A.E. Mark, H.J. Berend sen, GROMACS: fast, flexible, and free, Journal of computational chemistry 26 (2005) 1701-1718.

[25] O. Vang, N. Ahmad, C.A. Baile, J.A. Baur, K. Brown, A. Csiszar, D.K. Das, D. Delmas, C. Gottfried, H.Y. Lin, Q.Y. Ma, P. Mukhopadhyay, N. Nalini, J.M. Pezzuto, T. Richard, Y. Shukla, Y.J. Surh, T. Szekeres, T. Szkudelski, T. Walle, J.M. Wu, What is new for an old molecule? Systematic review and recommendations on the use of resveratrol, PloS one 6 (2011) e19881.

[26] T. Walle, F. Hsieh, M.H. DeLegge, J.E. Oatis, Jr., U.K. Walle, High absorption but very low bioavailability of oral resveratrol in humans, Drug Metab Dispos 32 (2004) 1377-1382. 
Figures:
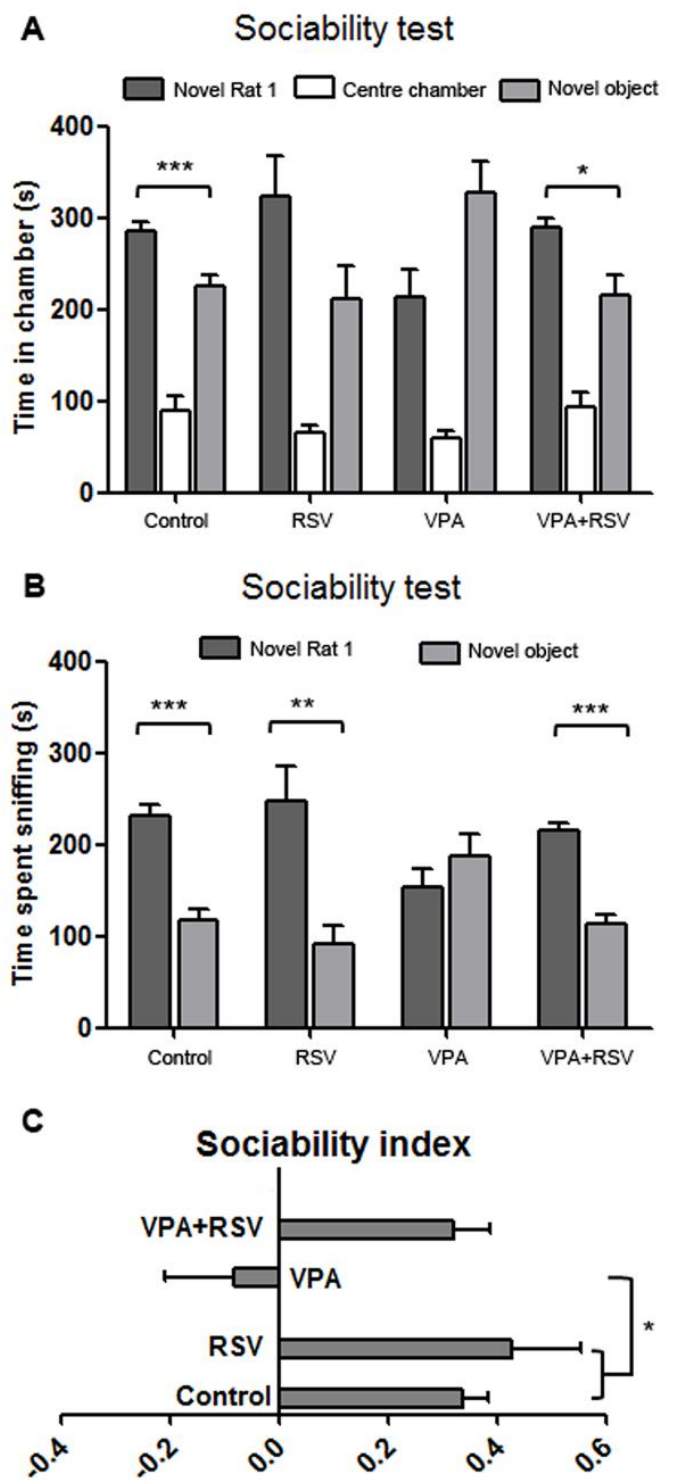

Figure 1. RSV administration prevents the sociability deficit present in the VPA model of autism in rats. After 5 minutes of acclimatization, male rats were allowed to explore all chambers for 10 min. (A) Time spent in chambers. (B) Time spent exploring novel rat 1 or novel object. (C) Sociability index. ${ }^{*} p<0.05 .{ }^{*}$ $p<0.01,{ }^{* * *} p<0.001 . n_{\text {Control }}=7, n_{R S V}=7, n V P A=19, n V P A+R S V=10$. 

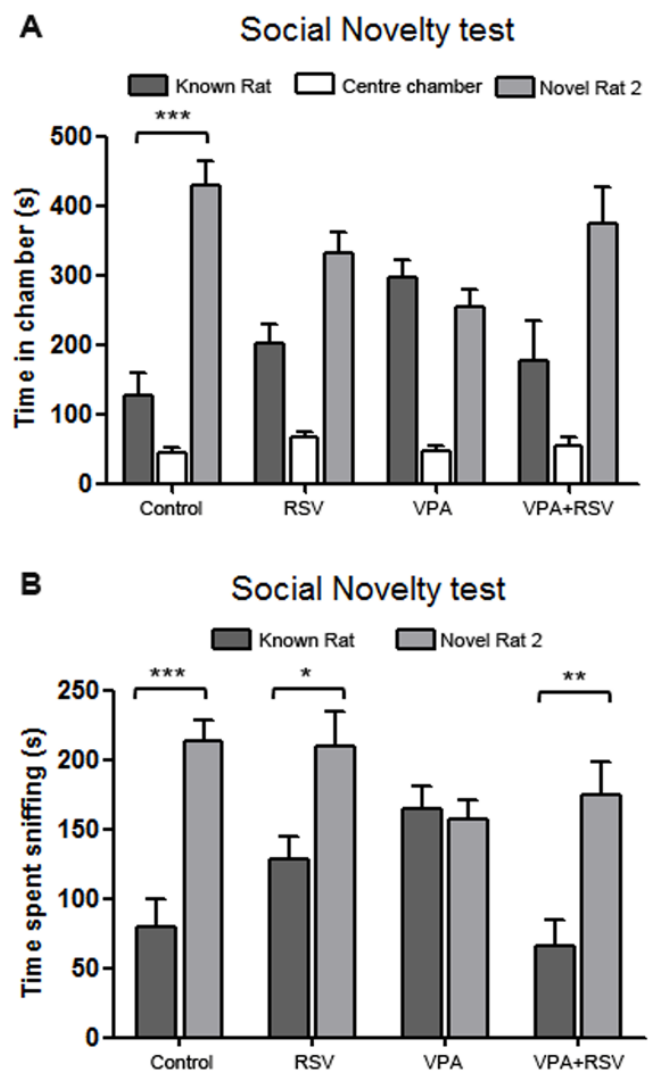

C Social Novelty Preference index

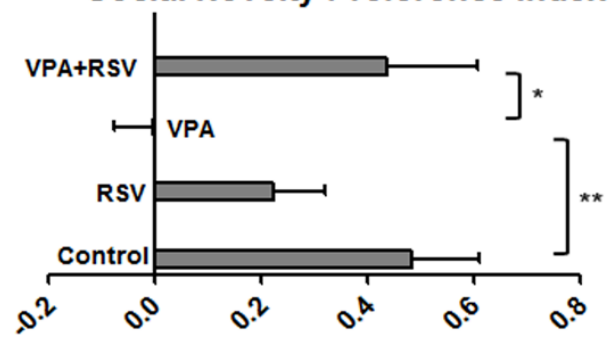

Figure 2. Prenatal RSV averts VPA detrimental effect on social novelty

preference. Immediately after the sociability test, a 10-minute test was performed in the three-chambered apparatus. (A) Time spent in chambers. (B) Time spent exploring the rats. (C) Social Novelty Preference index. ${ }^{*} p<0.05 .{ }^{* *} p<0.01,{ }^{* * *}$ $\mathrm{p}<0.001 . \mathrm{n}_{\text {Control }}=7, \mathrm{n}_{\mathrm{RSV}}=7, \mathrm{n}_{\mathrm{VPA}}=12, \mathrm{n}_{\mathrm{VPA}}+\mathrm{RSV}=9$ 

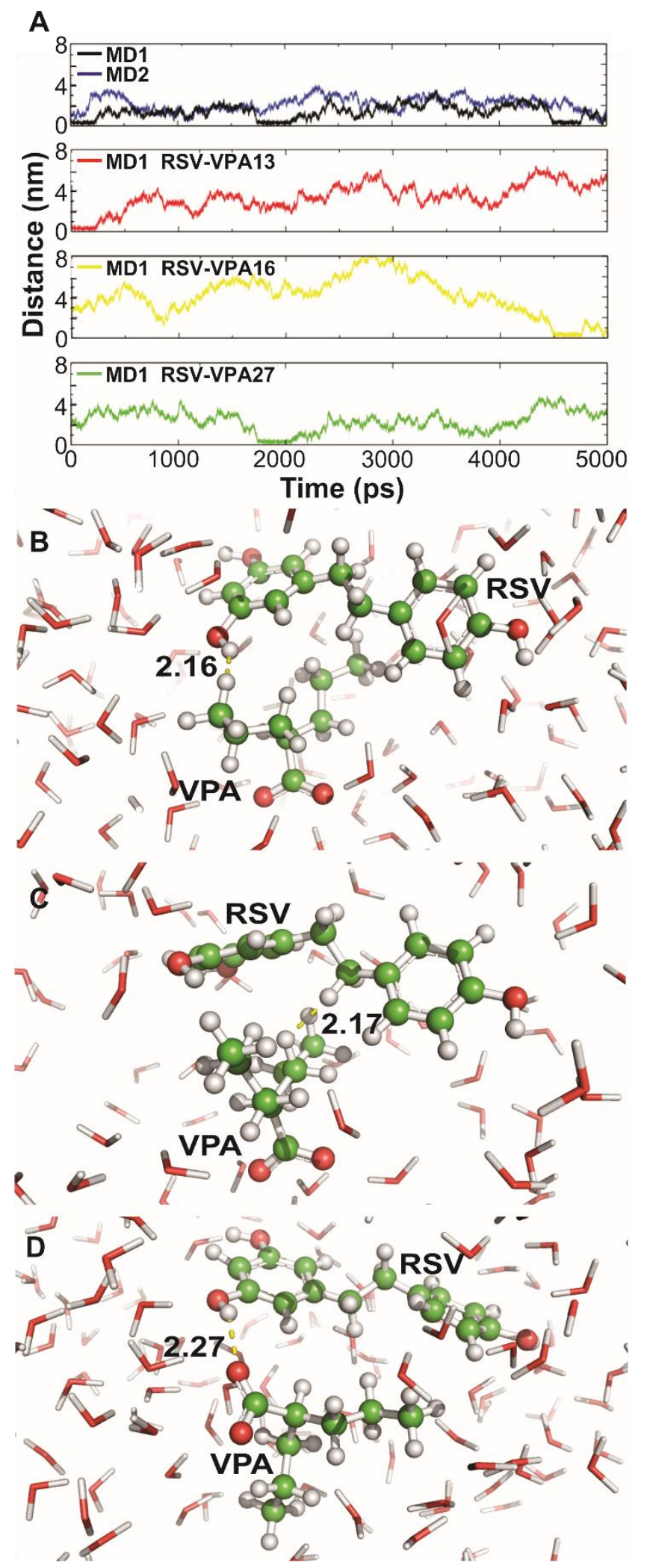
Figure 3. Representative data of interaction distances as result of molecular dynamics simulations. (A) First box represents the minimum distances between RSV and the closest molecule of VPA (any molecule) during two distinct simulation experiments (blue or black line). Graphs representing the distances of three isolated VPA molecules to RSV are shown in the second, third and fourth boxes. (B) Spatial orientation of VPA13 and RSV during approximation at 100ps (MD1). (C) Spatial orientation of VPA27 and RSV during approximation at $1916 \mathrm{ps}$ of simulation (MD1). (D) Spatial orientation of VPA16 and RSV during approximation at $4600 \mathrm{ps}$ of simulation (MD1) 